\title{
Study of propagation of harmful factors of fire in short road tunnels with different inclinations
}

\author{
Nicolae Ilias ${ }^{1}$, Omar Lanchava ${ }^{2,3}$, Giorgi Nozadze ${ }^{2,3}$, and David Tsanava ${ }^{3}$ \\ ${ }^{1}$ University of Petrşani, Petroşani, Universităţii Street 20, Romania \\ ${ }^{2}$ G. Tsulukidze Mining Institute, Tbilisi, Mindeli Street 7, Georgia \\ ${ }^{3}$ Georgian Technical University, Tbilisi, Kostava Street, 77, Georgia
}

\begin{abstract}
The paper considers the spread of combustion products caused by fires with different heat release rates: $5,10,15,20,30,50 \mathrm{MW}$ in up to $400 \mathrm{~m}$ long tunnels. The slope of the tunnels on numerical models is - 0,1 , $3,5,7,9 \%$. The cross-sectional area of the tunnel is $42.5 \mathrm{~m}^{2}$. The paper describes the dynamic variability of damaging factors caused by the "chimney effect" such as carbon monoxide and temperature. Modeling was done with FDS software by using the finite volume method. The time of process modeling is $180 \mathrm{~s}$. The minimum cell size of the finite volume is $0.25 \times 0.25 \times 0.25 \mathrm{~m}$. The hearth of fire is in the central part of the tunnel. The obtained results are given in the plane of the central longitudinal section of the tunnel. The boundary condition is given as an increment of the dynamic pressure caused by the height difference between the portals in normal conditions. The dependence of fire-induced backlayering distance on the fire heat release rate and the increment of the dynamic pressure caused by tunnel inclination are studied. The theoretical results and those obtained by modeling of the backlayering distance and velocity are analyzed and compared. The locations of high-risk factors for each damaging factor along the tunnel are identified. Their quasi-stationary nature is described and the intervals of transient processes typical to each factor are determined in the course of modeling. It is advisable to use the obtained results to respond to emergencies caused by fire in relevant tunnels.
\end{abstract}

\section{Introduction}

More than 50 motor tunnels must be built in Georgia in the nearest 3 or 5 years and as their design solutions suggest, the only risk-factor of the expected threat is fire. The results of the study give the worst scenarios of the spread of harmful combustion factors typical to longitudinal ventilation. In this case, the fresh air inlet is located hypsometrically higher than the fire hearth is. Consequently, the asymmetry of the spread of combustion products towards the fire hearth what is a common typical feature of this process, affects safety. The essence of the problem is to determine the important technological parameters of regularities of variation of air current aerodynamics and ventilation in a tunnel under the impact of fire for different tunnel gradients, heat release rate, tunnel cross section, ratio of the tunnel width to height and tunnel fill coefficient by transport and in terms of complicated tunnel aerodynamics due to extra barriers in the tunnel. 
This is a common problem in the world. By considering the strong fires in the tunnels of the world, the European Union paid particular attention to the Trans-European Network (TEN) recognizing the safety of the existing and planned tunnels as a priority. For the TEN tunnels with their length exceeding $500 \mathrm{~m}$, the European Parliament and the European Council issued Directive EC2004/54 regarding the minimum level of safety. Under the Directive and expert opinions, fire safety expenses for road tunnels in the EU countries vary between $€ 2.6-6.3$ billion. $€ 2.6$ billion is needed for the ventilation systems, while their improvement technology associated with fewer expenses $[1,2]$.

We have studied and presented the critical velocity variability according to the tunnel slope, the grade correction factor of this variability, the nature of spread of smoke and temperature distribution. The spread of smoke is specified according to the tunnel length and the distribution of temperature and carbon monoxide is described 1-2 $\mathrm{m}$ above the tunnel floor. The nature of variability of these values is given as diagrams.

The obtained results are applicable in emergency response projects, as well as in designing common and emergency ventilation and training rescuers and firefighters to improve their skills.

\section{Theory}

In order to specify the terms, let us consider some major characteristics typical to fire [3].

The convective heat released by fire is defined by formula

$$
\phi_{c}=\rho_{0} \epsilon_{p} \tau_{G} A \Delta T,
$$

The definitions in formula (1) and those given below are explained in Table 1.

Table 1. Definition and dimensions of the values given in the formulas.

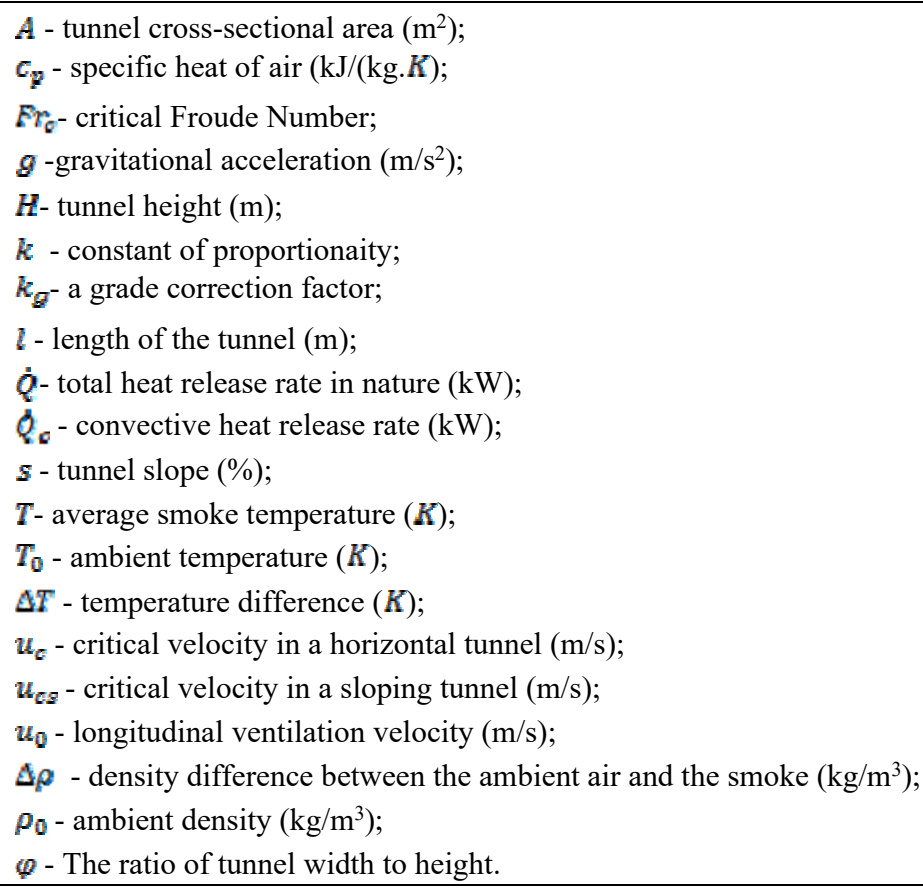


The dependence between the convective heat and total heat is as folows:

$$
\hat{Q}_{c}=0.7 \hat{Q},
$$

Average smoke temperature is calculated with formula>

$$
T=T_{0}+\frac{\phi_{\varepsilon}}{Q_{Q} C_{p} A M_{\varepsilon}},
$$

Thomas admitted [4] that the combustion products are evenly distributed in the air at the hearth of fire and that the ventilation velocity must exceed the rate of spread of the combustion products to prevent backlayering. As it is known, the combustion products spread due to their low density, and the type of motion of the air current will also depend on the ratio of inertia and ejection forces in it. The ratio of these forces is determined by the critical Froude Number. In this case, the ejection force is common for smoke, and the inertia force is common for the fresh air current. This assumption is known as the Critical Froude Model, which uses critical Froude Number equal to 4.5, is the most well-known model related to the given problem and is widely used in engineering.

$$
F r_{c}=\frac{\Delta \rho g H}{R_{Q} u_{Q}^{2}},
$$

One of the most important parameters of fire - the critical velocity for horizontal tunnels is generally defined by formula:

$$
u_{c}=k\left(\frac{s Q_{c} H}{\rho_{0} C_{p} F A}\right)^{1 / 3}
$$

Constant of proportionality in formula (5) is defined by critical Froude Number>

$$
k=F_{c}{ }^{-1 / 3},
$$

Based on the results of the numerical experiments, the numerical values of a grade correction factor, another important parameter of a fire dependant on the tunnel inclination, may be defined by formula

$$
k_{g}=\frac{w_{a s}}{w_{c}}
$$

The third important factor, the backlayering length is defined by formula

$$
\frac{\Sigma_{b}}{H}=18.5 \ln \left(\varepsilon_{c s} / w_{c}\right)
$$

The present work gives the calculation of the critical velocity when the basis is the numerical modeling results. The gained final results are given alongside with the regularities of the temperature and carbon monoxide distribution as diagrams.

\section{Results and analysis of numerical experiments}

The article considers the spread of combustion products caused by fires with different heat release rates: $5,10,15,20,30,50 \mathrm{MW}$ in up to 400 -m-long tunnels. The slope of the tunnels on numerical models is $-0,1,3,5,7,9 \%$. The cross-sectional area of the tunnel is $42.5 \mathrm{~m}^{2}$. The paper describes the dynamic variability of damaging factors caused by the "chimney effect" such as carbon monoxide and temperature. Modeling was done with FDS software by 
using the finite volume method. The time of process modeling is $180 \mathrm{~s}$. The minimum cell size of the finite volume is $0.25 \times 0.25 \times 0.25 \mathrm{~m}$ and the maximum size is $0.5 \times 0.5 \times 0.5 \mathrm{~m}$ [5]. The hearth of fire is in the central part of the tunnel. Polyurethane M 27 was used as a combustion agent for fire modeling; the variability of the area of the fire combustion surface in numerical experiments was $5-10 \mathrm{~m}^{2}$. The fire hearth was located $0.6 \mathrm{~m}$ from the floor.

The validity of the numerical experiments was determined according to the numerical value of the critical velocity. In particular, in the course of the experiments, the critical velocity was calculated according to the maximum velocity of the frontal spread of the combustion products at the fire hearth, what corresponds to $20 \mathrm{~s}$ of modeling time (Fig. 1). As the same figure shows, in the course of modeling, i.e., depending on the tunnel length, the frontal velocity of the combustion products decreases significantly as a result of the tunnel aerodynamic resistance. Therefore, the maximum numerical values of velocities given in Fig. 1 are the critical velocities for horizontal tunnels for different fire heat release rate.

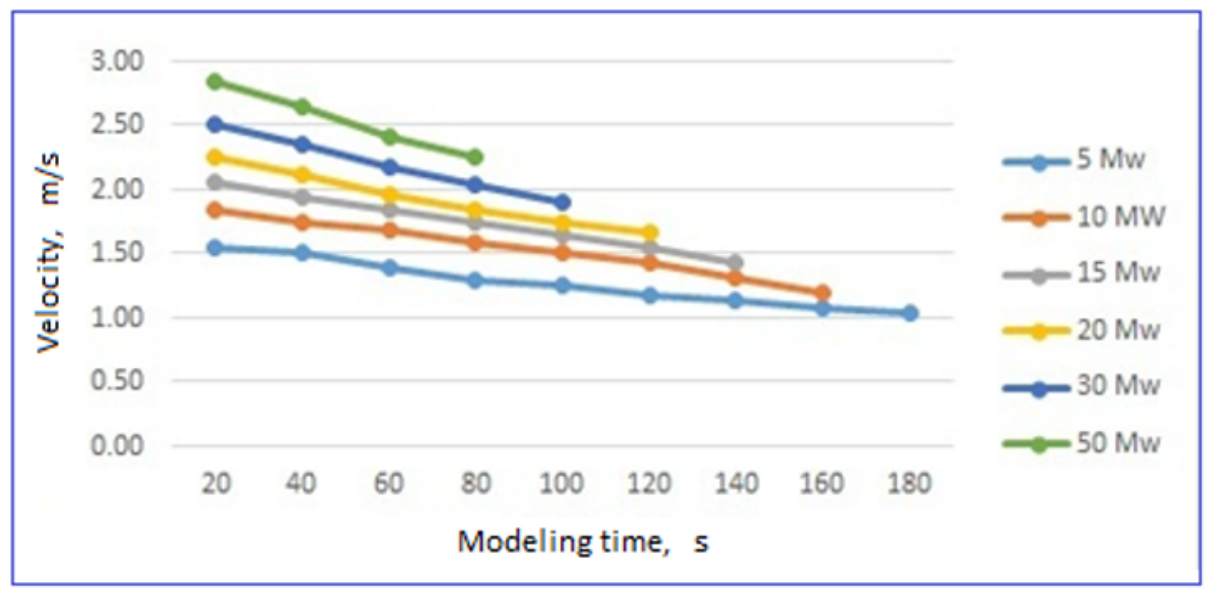

Fig. 1. Variability of the rate of spread of combustion products in a horizontal tunnel in the numerical simulation when the rate of fire heat release is variable in terms of natural ventilation conditions.

The critical velocity depending on the fire heat release rate was calculated with formula (4) for condition $\mathrm{Fr}_{\mathrm{c}}^{-1 / 3}=0.606$. The results of experiments and theory are given in Table 2.

Table 2. Comparison of the theoretical and numerical results of the critical velocity.

\begin{tabular}{|c|c|c|c|c|c|c|}
\hline $\begin{array}{c}\text { Fire heat release rate, } \\
\dot{Q}, \mathrm{MW}\end{array}$ & $5 \mathrm{MW}$ & $10 \mathrm{MW}$ & $15 \mathrm{MW}$ & $20 \mathrm{MW}$ & $30 \mathrm{MW}$ & $50 \mathrm{MW}$ \\
\hline $\begin{array}{c}\text { Critical velocity, } \\
u_{c}, \mathrm{~m} / \mathrm{s} \text { experimental }\end{array}$ & 1.55 & 1.85 & 2.05 & 2.25 & 2.5 & 2.85 \\
\hline $\begin{array}{c}\text { Critical velocity, } \\
u_{c}, \mathrm{~m} / \mathrm{s}, \text { theoretical }\end{array}$ & 1.75 & 2.30 & 2.60 & 2.77 & 2.87 & 2.89 \\
\hline Relative error \% & 11.4 & 19.6 & 21.1 & 18.7 & 13 & 1.4 \\
\hline
\end{tabular}

Although from an engineering point of view, the results of the theoretical and experimented tests coincide with one another sufficient accuracy to be applicable in practice, it is noteworthy that the relative error for the interval of the fire heat release rates given by Table 2 acquires minimum values at the limits of the interval. This fact indicates that additional factors must be considered in the method to determine the backlayering velocity, what must be related to the nature of the variability of the Froude number in the said interval, 
as well as the peculiarities of the variability of the temperature and average density of the air mixture at the hearth of fire.

The results of the numerical tests planned by us allow obtaining the information about the qualitative and quantitative trends of distribution of two main harmful factors - the temperature and carbon monoxide, to be used in practice. For this purpose, below we give the dynamics of temperature propagation in the uphill and downhill flows in the tunnels with different inclinations when the hearth of fire is in the center of the tunnel. In this experiment, the natural value of the dynamic pressure resulting from the difference in heights between the portals, known in the literature as the "chimney effect", was obtained through modeling the natural conditions at the portals. Posing the problem in this manner facilitates the realization of the boundary condition problem at the same time maintaining the generality of the problem.

Figure 2 shows the process of calculation of the temperature distribution with FDS software. Fig. 3 shows the dynamics of the temperature distribution in the uphill flow along the tunnel, and Fig. 4 shows the variability of the same value in the downhill flow. The averaged numerical values of temperature are given along the longitudinal symmetry axis of the tunnel, depending on the modeling results. The numerical values are calculated within the hypsometric range of the tunnel height on the vertical plane, at the height of 1-2 $\mathrm{m}$ from the floor of the tunnel. The vertical plane itself is located along the axis of the given longitudinal symmetry. For demonstration purposes, the given vertical plane on 3D tunnel model is given in blue (see Figure 2).

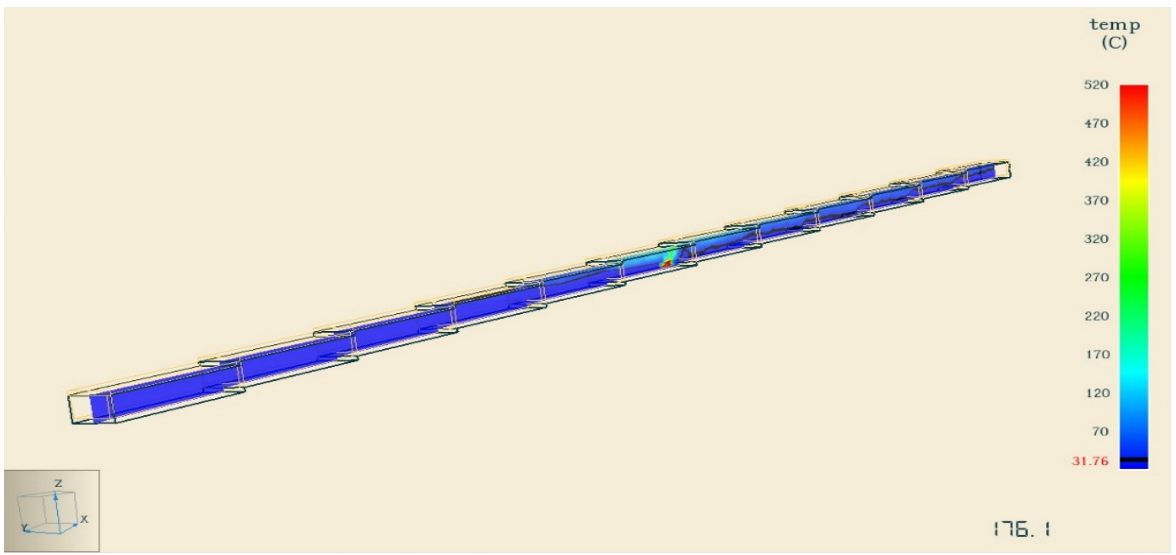

Fig. 2. Calculation of the temperature distribution with FDS software for 3D model. The vertical plane running along the tunnel axis, on which the temperatures were averaged.

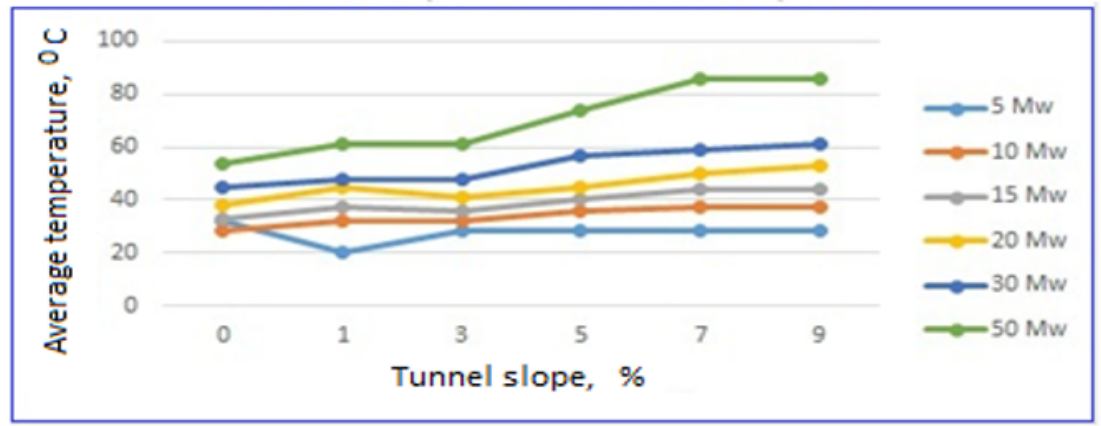

Fig. 3. The variability of the mean temperature for the uphill flow near the upper portal depending on the tunnel slope. 


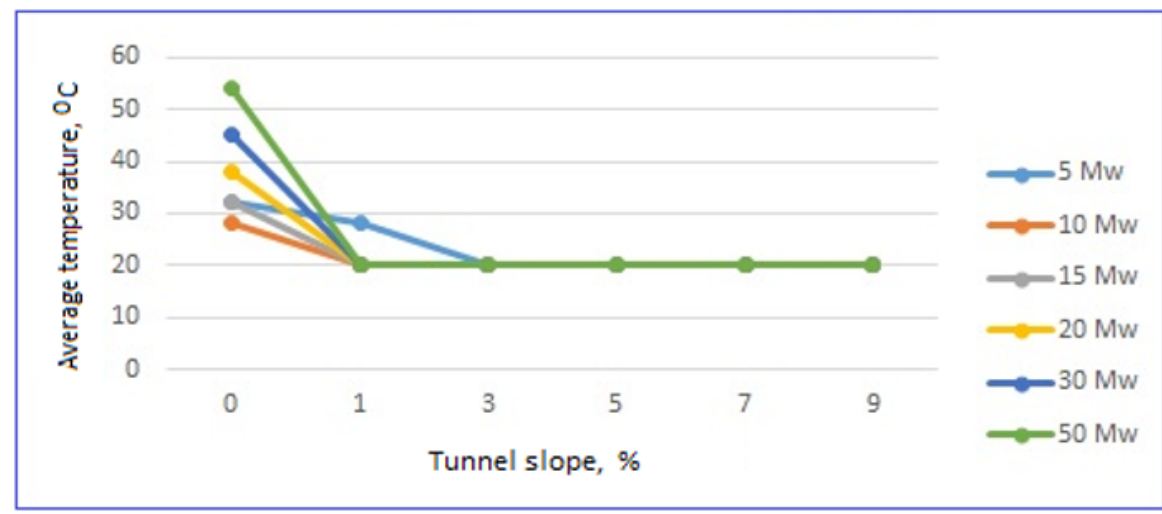

Fig. 4. The variability of the mean temperature for the downhill flow near the lower portal depending on the tunnel slope.

The calculation results evidence that in the uphill flow, the temperature from the fire hearth increases rapidly to reach the level inconsistent with the human's vital functions ( $T>60^{\circ} \mathrm{C}$ ) [6]. The given level increases in a directly proportional manner depending on the fire heat release rate and tunnel slope.

At the same time, we would like to note that as the fire strength increases, the quasistationary turbulent flow establishes very quickly (in approximately $90-100 \mathrm{~s}$ ) and the picture of the temperature distribution no longer changes during the remaining modeling time.

A qualitatively opposite picture in view of temperature distribution is observed in the downhill flow from the fire hearth. In this case, the dynamic pressure increment occurring between the portals by the "chimney effect" acts opposite to the traction induced by fire.

Therefore, it leads to a similarly rapid stabilization of the backlayering process. As a result, the transient processes in the downhill flow end in virtually the same time and the process of rapid establishment of the temperature at the entrance of the portal practically up to the hearth of fire occurs.

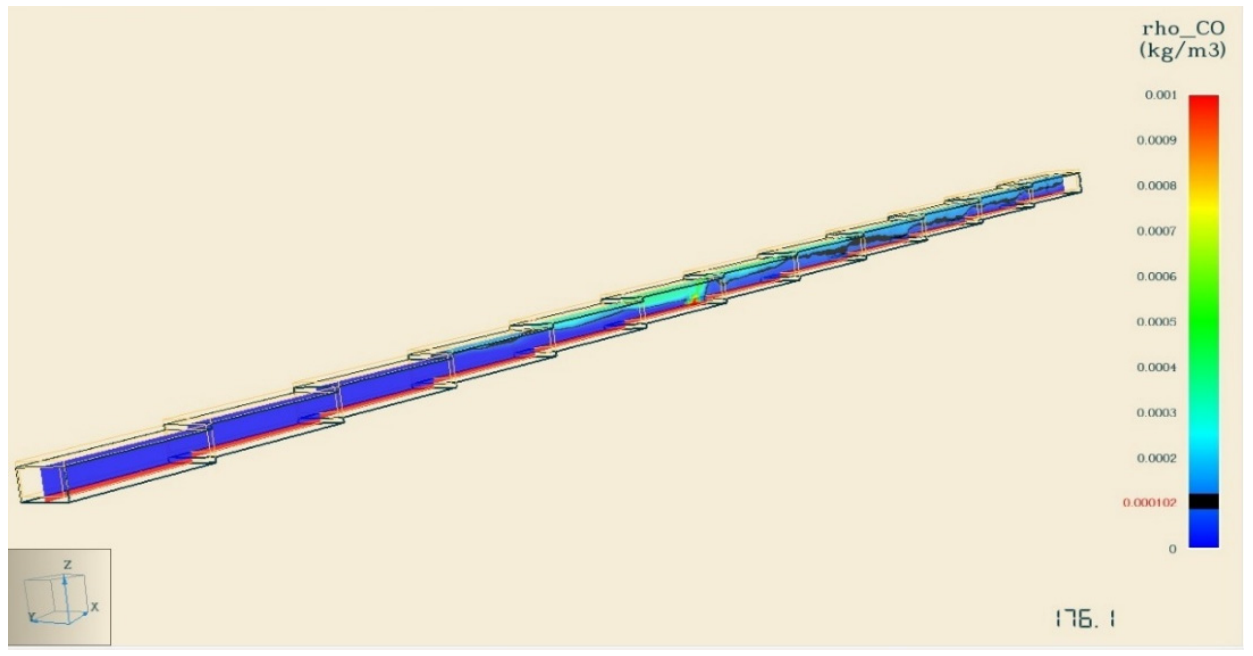

Fig. 5. Calculation of carbon monoxide with FDS software with a 3D model. Blue color denotes the vertical plane running on the tunnel axis, on which the carbon monoxide densities were averaged. 
No less harmful factor is the distribution of the carbon monoxide concentration on both sides of the fire hearth. It should be noted that in this case too, the qualitative picture does not change. The difference is that the density of carbon monoxide varies within quite a wide range in the tunnel, within the concerned hypsometric interval, distanced from the tunnel floor by $1-2 \mathrm{~m}$.

Figure 5 shows the process of numerical modeling as a 3D illustration of the dynamics of the distribution of carbon monoxide.

The dynamics of carbon monoxide distribution in the uphill flow along the tunnel is given in Figure 6, while Figure 7 shows the variability of the same value in the downhill flow. In this case too, the averaged numerical values are given on the longitudinal symmetry axis of the tunnel and the numerical values are calculated on the vertical plane within the hypsometric interval of the tunnel height, 1-2 $\mathrm{m}$ high from the tunnel floor.

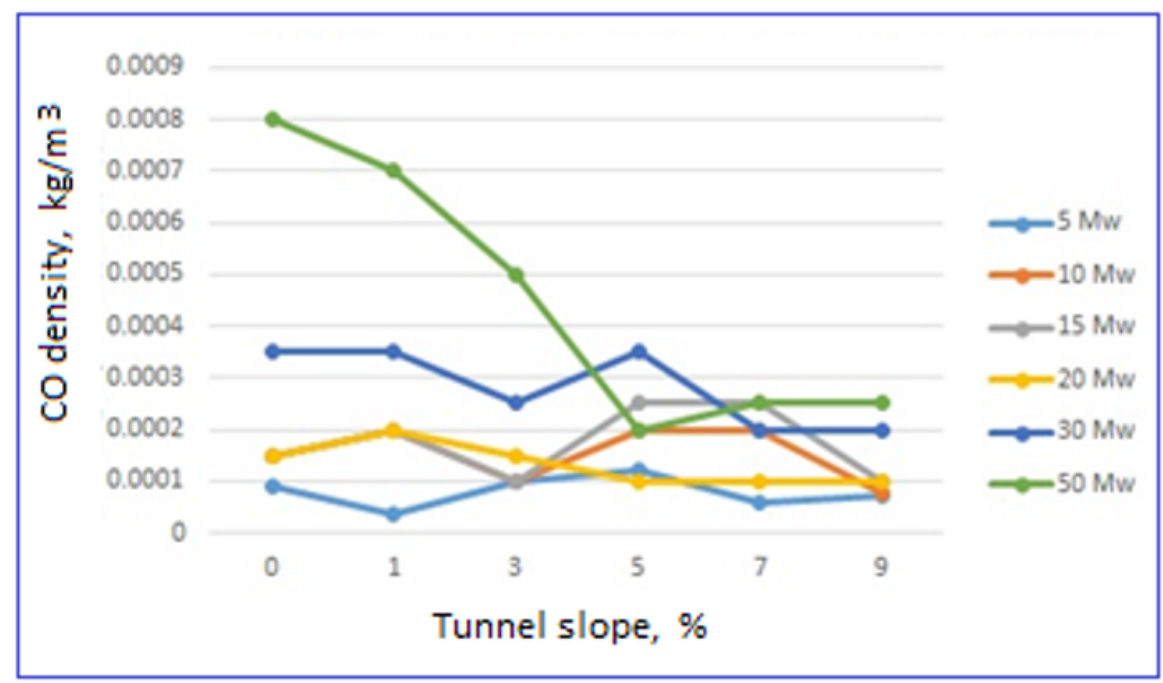

Fig. 6. Variability of the average density of carbon monoxide in the uphill flow depending on the tunnel slope near the upper portal.

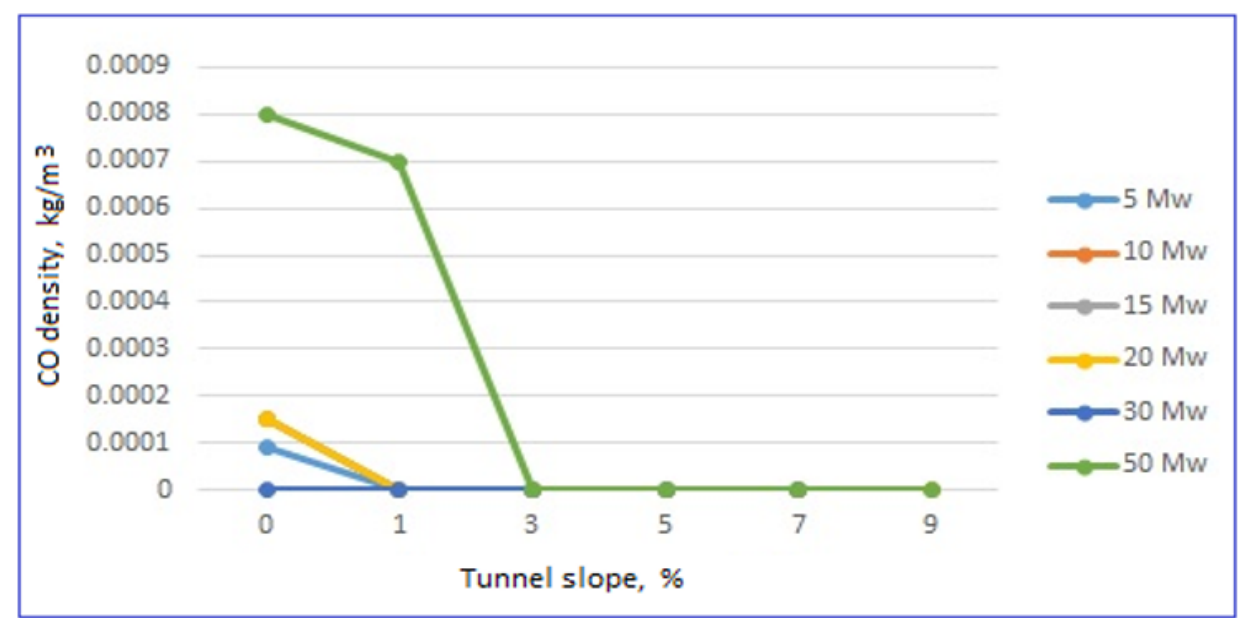

Fig. 7. Variability of the average density of carbon monoxide depending on the tunnel slope in the downhill flow at the lower portal. 
The picture of carbon monoxide distribution results from the dynamic pressure difference induced by the "chimney effect" and the interaction of the currents induced by the fire heat release rate. The presented results show that the dynamic pressure difference for 5-15 MW fires at the portals does not have a great impact on the variability of the density of carbon monoxide in the uphill flow because of the low concentration of carbon monoxide at the hearth of fires with the given heat release rate. The outcomes for 30 and $50 \mathrm{MW}$ fires are different, since in this case, the effect of ventilation is much stronger for very sloping tunnels.

The presented results show that the downhill flows formed by the dynamic pressure difference between the portals in the tunnels with all possible slopes considered, similarly to the temperature, rapidly stabilize the processes of distribution and practically relieve the given branch off the increase in the carbon monoxide density, what at one glance, is unexpected under the impact of backlayering caused by fire. So, the given harmful factor is also relevant for low-slope tunnels of a descending branch during the backlayering processes.

\section{Conclusion}

The present paper explores the dynamic variability of the spread of such harmful factors, as carbon monoxide and temperature, with FDS software and full-scale model, by applying the finite volume method in terms of : 5, 10, 15, 20, 30, $50 \mathrm{MW}$ fires. In the numerical simulation, the cross-sectional area of the tunnel was $42.5 \mathrm{~m}^{2}$ and the tunnel slope was $0,1,3,5,7,9 \%$. The time of process modeling was $180 \mathrm{~s}$. The minimum cell size of the finite volume was $0.25 \times 0.25 \times 0.25 \mathrm{~m}$. The hearth of fire is in the central part of the tunnel. The obtained results are given in the vertical plane given on the central axis of the tunnel, within 1-2 m hypsometric interval from the tunnel floor.

The obtained results evidence that in the uphill flow, the temperature from the fire hearth increases rapidly to reach the level inconsistent with the human's vital functions $\left(T>60^{\circ} \mathrm{C}\right.$ ) [6]. The given level is characterized by a directly proportional increase depending on the fire heat release rate and tunnel slope.

It should be noted that as the fire heat release rate increases, a quasi-stationary turbulent flow establishes very quickly (in approximately 90-100 s) and the picture of the temperature distribution no longer changes during the remaining modeling time.

A qualitatively opposite picture from the view of temperature distribution is observed in the downhill flow from the fire hearth. In this case, the dynamic pressure increment occurring between the portals by the "chimney effect" acts opposite to the traction induced by fire. Therefore, it leads to a similarly rapid stabilization of the backlayering process. As a result, the transient processes in the downhill flow end in virtually the same time and the process of rapid establishment of the temperature at the entrance of the portal practically up to the hearth of fire takes place.

The presented results show that the dynamic pressure difference for 5-15 MW fires at the portals does not have a great impact on the variability of the density of carbon monoxide in the uphill flow as a result of the low concentration of carbon monoxide at the hearth of fires with the given heat release rate. The outcomes for 30 and $50 \mathrm{MW}$ fires are different, since at this time, the effect of ventilation is much stronger for very sloping tunnels.

This work was supported by Shota Rustaveli National Science Foundation (SRNSF) [Grant number AR-19-1936, Project title "Development and testing of transformable system to save life in road tunnel in case of fire"]. 


\section{References}

1. Directive TRANS/AC7/9 Economic Commission for Europe (2001)

2. D. Theologitis, Euro transport 3, 16 - 22 (2005)

3. Y.Z. Li, and H. Ingason, Fire Safety Journal 99, 22-26 (2018)

4. P.H. Thomas, Fire Research Notes 351, Available on http://www.iafss.org/publications/frn/351/-1

5. O. Lanchava, N. Ilias, G. Nozadze, S.M. Radu, R.I. Moraru, Z. Khokerashvili, and N. Arudashvili, Environmental Engineering and Management Journal18 (4), 317-325 (2019)

6. O. Lanchava, G. Nozadze, N. Bochorishvili, Z. Lebanidze, N. Arudashvili, M. Jangidze, and K. Tsikarishvili," Transport Bridge Europe-Asia" Materials of International Conference, 29-34 (2014) 